Conclusions: There was no difference in early major morbidity and long term growth and neurodevelopmental outcome between SGA and AGA VLBW infants.

1195

\section{STATISTICAL EVALUATION OF STREPTOCOCCUS RESISTANCE TO ANTIBIOTICS IN CHILDREN}

S.I. Iurian ${ }^{1,2}$, S. Iurian ${ }^{2,3}$, M.L. Neamtu ${ }^{1,2}$, B.M. Neamtu' ${ }^{1,2}$, B.I. Mehedintu ${ }^{1,2}$

${ }^{1}$ Lucian Blaga University, ${ }^{2}$ Pediatric Clinic, ${ }^{3}$ Clinical Laboratory, Sibiu, Romania

Backgroundandaims: The „EuropeanSurveillance” reported resistance of group A streptococci to macrolides as follows: $11 \%$ of isolates in Portugal and $32 \%$ in Italy. First clindamycin-resistant isolate was reported in 1999. The authors evaluated antibiotic resistance of pyogenic streptococci (serogroups A, C, G) for hospitalized children.

Methods: There were realised 714 throat cultures during 30 days period: 56 isolates $(7,84 \%)$ were positive (large-colony streptococci). Inclusion criteria (antibiogram performing): beta-hemolytic streptococci group A (GAS), C (GCS) and G (GGS). Exclusion criteria: small-colony streptococci. 34 isolates were selected for diffusimetric method (Mueller Hinton medium 5\% sheep blood; $S$. pneumoniae ATCC 49619 strain as quality control) using penicillin, erythromycin and clindamycin disks. The cases were reevaluated after 10 days penicillin therapy.

Results: 7 strains $(20,58 \%)$ were erythromycin resistant (5 strains GAS, 1 GCS, 1 GGS), 2 strains $(5,88 \%)$ clindamycin resistant beta-lactamases produced by (1 strain GAS,1 GGS). One case wasn't cured after penicillin therapy (antibiogram revealed resistance just for erythromycin), justifying Clindamycin treatment with good evolution. The other cases were successfully treated with penicillin.

\section{Conclusions:}

1.The study confirmed macrolides resistance for $1 / 5$ strains of streptococci pyogenes;

2. It isn't recommended routine antibiogram;

3. Clindamycin resistance was identified for $6 \%$ strains;
4. Penicillin is first choice treatment for streptococcal pharyngitis;

5. One case was penicillin resistant, probably due to bacterial adaptive mechanisms: the streptococcus „internalisation” or saprophytic bacteria from mouth or streptococcus persistance in lingual tonsils;

6. The persistence of Streptococcus in pharynx after penicillin treatment is different from concept of GAS resistance to penicillin.

\section{6}

\section{EPIDEMIOLOGICAL ASPECTS OF SALMONELLA AND SHIGELLA INFECTIONS IN CHILDREN. STATISTICS OF PEDIATRIC CLINIC}

S.I. Iurian ${ }^{1,2}$, S. Iuriann, ${ }^{2,3}$, V. Bunescu², B.M. Neamtu ${ }^{1,2}$, B.I. Mehedintu ${ }^{1,2}$

${ }^{1}$ Lucian Blaga University, ${ }^{2}$ Pediatric Clinic, ${ }^{3}$ Clinical Laboratory, Sibiu, Romania

Background and aims: Prevalence of Salmonella and Shigella infections varies due to inconsistent diagnosis techniques. Authors evaluate Salmonella I Shigella infections from epidemiological and evolution points of view for hospitalized children.

Methods: There were performed 585 stool cultures during 3 months period using mediums (agardesoxycholate-citrate-lactose medium, selenite broth), biochemical tests (mobility-indol-urease, triple-sugar-iron), latex agglutination, serological somatic (O) Salmonella tests. Mueller-Hinton medium was chosen for diffusimetric antibiogram using disks for ampicillin(AMP), ceftazidime(CAZ), trimetoprim-sulfametoxazol(SXT), nalidixic acid(NA) and colimycin(CT). Inclusion criteria for antibiogram: newly diagnosed Salmonella / Shigella cases. Exclusion criteria: positive stool culture for previously treated children.

Results: From 585 samples, 39 (6,67\%) were positive: 34 Salmonella isolates (17 group B, 15 group D, 2 group C) and 5 Shigella isolates (4 samples $S$. sonnei, 1 sample $S$. boydii). From Salmonella samples, 13 represented posttherapeutic relapses ( 5 group B, 7 group D, 1 sample group $\mathrm{C}$ ). There were performed 21 antibiograms: group B Salmonella -from 9 samples, 6 strains AMP resistant, 2 SXT resistant; group D Salmonella -from 7 samples, 2 strains NA resistant; group C Salmonella -1 susceptible strain; Shigella sonnei -3 AMP and SXT resistant strains; Shigella boydii -1 